\title{
Etiología bacteriana de infecciones oculares externas
}

\author{
Patricia Hernández Rodríguez M.Sc $\quad{ }^{1}$ y Gladys Quintero M.Sc. $\quad{ }^{2 *}$ \\ ${ }^{1}$ Facultad de Optometría, Departamento de Ciencias Básicas, Universidad de la Salle y Pontificia Universidad Javeriana. Bogotá \\ Colombia*, ${ }^{2}$ Departamento de Ciencias Básicas. Universidad de la Salle y Facultad de Ciencias de la Salud, Programa de \\ Bacteriología, Universidad Colegio Mayor de Cundinamarca. \\ Recibido: 01-08-03; Aceptado: 23-10-03
}

\section{RESUMEN}

En esta investigación fueron evaluados 286 pacientes que presentaron conjuntivitis bacteriana como impresión diagnóstica más frecuente. En 286 cultivos microbiológicos realizados se obtuvieron 177 aislamientos bacterianos, encontrándose un 73.45\% de flora Gram positiva siendo las especies más frecuentes S. epidermidis (48.46\%), S. aureus (35.38\%),S. pneumoniae (4.61\%) y Corynebacterium sp $(2.31 \%)$. El 26.55\% correspondió a bacilos Gram negativos de los cuales el $74.47 \%$ son enterobacterias y el $25.53 \%$ microorganismos no fermentadores. En este estudio no se encontró diferencia significativa entre la convivencia o no con animales y tipo de microorganismo aislado asociado a zoonosis.

Palabras Claves: Infecciones oculares, S. aureus, S. epidermidis, C. meningosepticum, K. ascorbata, A. faecalis.

\section{ABSTRACT}

Bacterial etiology of external ocular infections in patients that live together or not with animals.

In this investigation 286 patients that presented bacterial conjunctivitis as more frequent diagnostic impression were evaluated. In 286 microbiological cultivations carried out 177 bacterial isolations were obtained, being $73.45 \%$ positive Gram flora being the most frequent species S. epidermidis (48.46\%), S. aureus (35.38\%), S. pneumoniae (4.61\%) and Corynebacterium sp. (2.31\%). 26.55\% corresponded to negative Gram bacilluses of which $74.47 \%$ is enterobacterias and $25.53 \%$ unfermented micro organisms. In this study was not found significant difference among the coexistence or not with animals and type of isolated micro organism associated to zoonosis.

Keywords: Ocular infections, S. aureus, S. epidermidis, C. meningosepticum, K. ascorbata, A. faecalis.

\section{INTRODUCCIÓN}

La patogénesis de las infecciones oculares está determinada por múltiples causas: la virulencia intrínseca del microorganismo, la respuesta natural del huésped y la estructura anatómica, fisiológica y bioquímica del ojo. Las infecciones pueden originarse externamente afectando las zonas directas de contacto; sin embargo, en ocasiones las bacterias afec-

\footnotetext{
*Correspondencia: Investigaciones@unicolmayor.edu.co
}

tan tejidos circundantes extendiendo la infección al ojo interno e incluso al cerebro (1).

A nivel ocular varios microorganismos generan infección. La mucosa conjuntival puede hospedar frecuentemente microorganismos como Staphylococcus epidermidiso Staphylococcus aureus; con menos frecuencia pueden recuperarse algunas Corynebacterias, Neisserias no patógenas y Streptococcus 
pyogenes. Estos microorganismos pueden ser causantes de diversas infecciones oculares, entre las cuales se encuentran: conjuntivitis, blefaroconjuntivitis, dacriocistitis, queratitis entre otras; además, dependiendo del tiempo de evolución de la infección y del tratamiento instaurado inicialmente, se pueden generar complicaciones severas que conducirían a pérdida parcial o total de la visión o incluso a septicemia.

Muchas enfermedades infecciosas son transmitidas por animales a humanos en condiciones naturales. El agente infeccioso determina la sintomatología clínica, que en las enfermedades de tipo zoonótico puede ser sistémica o localizada, afectando la piel, el ojo, el sistema nervioso, las vías respiratorias y gastrointestinales, entre otras (2).

A nivel ocular los microorganismos que generan infección, y que son transmitidos por animales de manera directa o indirecta, pueden ser bacterias como Francisella tularensis, Yersinia pestis, Pasteurella multocida, Chlamydia psittacii, Leptospira, Staphylococcus, y Streptococcus (3-7).

En Colombia, los estudios sobre infecciones oculares son limitados; por lo tanto, no se conoce la frecuencia de los microorganismos que causan estas patologías. Además, el diagnóstico, en la mayoría de los casos, es clínico: generalmente no se confirma con pruebas de laboratorio, lo cual genera que, en muchas ocasiones, especialmente en los casos de infecciones producidas por bacterias, el tratamiento no contrarreste la infección debido a que no se conoce con exactitud el agente etiológico implicado en la patología ocular. Por esta razón, este trabajo pretendió establecer qué tipo de bacterias producen infección en la población de estudio y evaluar si existe o no asociación de estos microorganismos con la convivencia o no con animales. Se estudiaron 286 pacientes que acudieron a consulta en el Instituto de Investigaciones Optométricas de la Universidad de La Salle y pacientes captados en brigadas de salud realizadas en veredas a las afueras de la ciudad de Bogotá. Cada paciente llenó un formulario que permitió recolectar la información básica. De todos los pacientes se emitió una impresión diagnóstica y en los casos de diagnóstico clínico presuntivo de infección ocular externa se tomaron muestras para diagnóstico bacteriológico, tinción de Gram y cultivo, con el fin de identificar el agente etiológico.

En los 286 pacientes se encontró como impresión diagnóstica más frecuente Conjuntivitis Bacteriana (34.61\%), Blefaritis Inespecífica (19.93\%) y Blefaritis Bacteriana (17.13\%). Se obtuvieron 177 aislamientos bacterianos, encontrándose un notorio predominio de flora Gram-positiva 73.45\%; las especies más frecuentes: Staphylococcus epidermidis (48.46\%), Staphylococcus aureus (35.38\%), Streptococcus pneumoniae $(4.61 \%)$ y Corynebacterium sp. (2.31\%). E1 26.55\% correspondió a bacilos Gram negativos de los cuales el $74.47 \%$ son enterobacterias y el $25.53 \%$ microorganismos no fermentadores.

Se aislaron algunos microorganismos hasta ahora no reportados en la literatura como agentes primarios de infección ocular (Enterococcus, Streptococcus grupo D no enterococo, Alcaligenes faecalis, Citrobacter sp, Chryseobacterium meningosepticum y Kruyvera ascorbata ). Es probable que al implementar el cultivo bacteriológico como apoyo diagnóstico en infección ocular, estos aislamientos sean más frecuentes y se pueda determinar su implicación como patógenos oculares.

En este estudio no se encontró diferencia significativa entre convivencia o no con animales y tipo de microorganismo aislado asociado a zoonosis. A partir de los resultados obtenidos en esta investigación se hace una contribución al estudio de las infecciones oculares en Colombia, ya que este es el primer trabajo sobre frecuencia de infecciones donde se relacionan los resultados microbiológicos con la convivencia o no con animales. Finalmente, los datos obtenidos de frecuencia relativa de infecciones oculares beneficia 
no sólo la salud visual de los pacientes sino también a los profesionales relacionados con esta área, otorgándoles herramientas que mejoran el diagnóstico y tratamiento de las mismas.

\section{Metodología}

286 pacientes, tanto del Instituto de Investigaciones Optométricas de la Universidad de la Salle, como captados en brigadas de salud, participaron de manera voluntaria y aprobaron la realización del estudio. Todos los pacientes fueron examinados con el fin de emitir una impresión diagnóstica y realizar la toma de muestra para confirmar el diagnóstico presuntivo. Los datos de los pacientes con diagnóstico clínico de infección ocular se obtuvieron mediante encuestas realizadas en el momento de la consulta; se llevó un registro de las mismas en un libro de pacientes, se creó una base de datos en el programa EPIINFO 6.04d y para establecer las relaciones entre las variables, se utilizó el programa ESTATA 6.0.

La muestra ocular fue tomada con un asa estéril desechable o con escobillón impregnado de carbón activado (según el caso); se tomaron muestras de la lesión y/o secreción, con el fin de realizar frotis para estudio microscópico por medio de la coloración Gram y fue transportada al laboratorio en medio de Stuart, el cual se mantuvo a temperatura ambiente hasta la inoculación en los medios de cultivo de aislamiento primario.

A partir del medio de transporte Stuart, la muestra fue cultivada en medios de aislamiento primario: agar sangre, agar chocolate y agar EMB. La identificación de especies se realizó de acuerdo al protocolo establecido para aislamiento de gérmenes comunes y se utilizó el ID BBL CRYSTAL para identificación de Gram negativos.

\section{Resultados}

El $56.30 \%$ de las 286 muestras procesadas fueron positivas. A partir de los 286 cultivos realizados se obtuvieron 177 aislamientos y se encontró que las especies se distribuyen según su frecuencia así: Staphylococcus epidermidis (35.60\%),Staphylococcus aureus (26\%), Eschericha coli (5.65\%), Streptococcus pneumoniae (3.39\%) y Enterococcus (3.39\%) (Tabla 1).

En 16 pacientes se aislaron dos tipos de microorganismos (Tabla 2).

Se realizó un análisis bivariado utilizando el programa ESTATA 6.0 con el fin de establecer una comparación entre los resultados microbiológicos y la convivencia o no con animales. De este análisis se observa que la presencia de microorganismos (55.37\%) es mayor con la convivencia con animales; sin embargo, no existe una diferencia significante (Tabla 3). En cuanto a los microorganismos aislados asociados a zoonosis, el 50\% (34/68) correspondió a Staphylococcus epidermidis, seguido de Staphylococcus aureus $35.30 \%(24 / 68)$ y, en menor porcentaje Streptococcus pneumoniae $(5,88 \%)$, Streptococcus viridans $(4,11 \%)$ y Streptococcus grupo D no

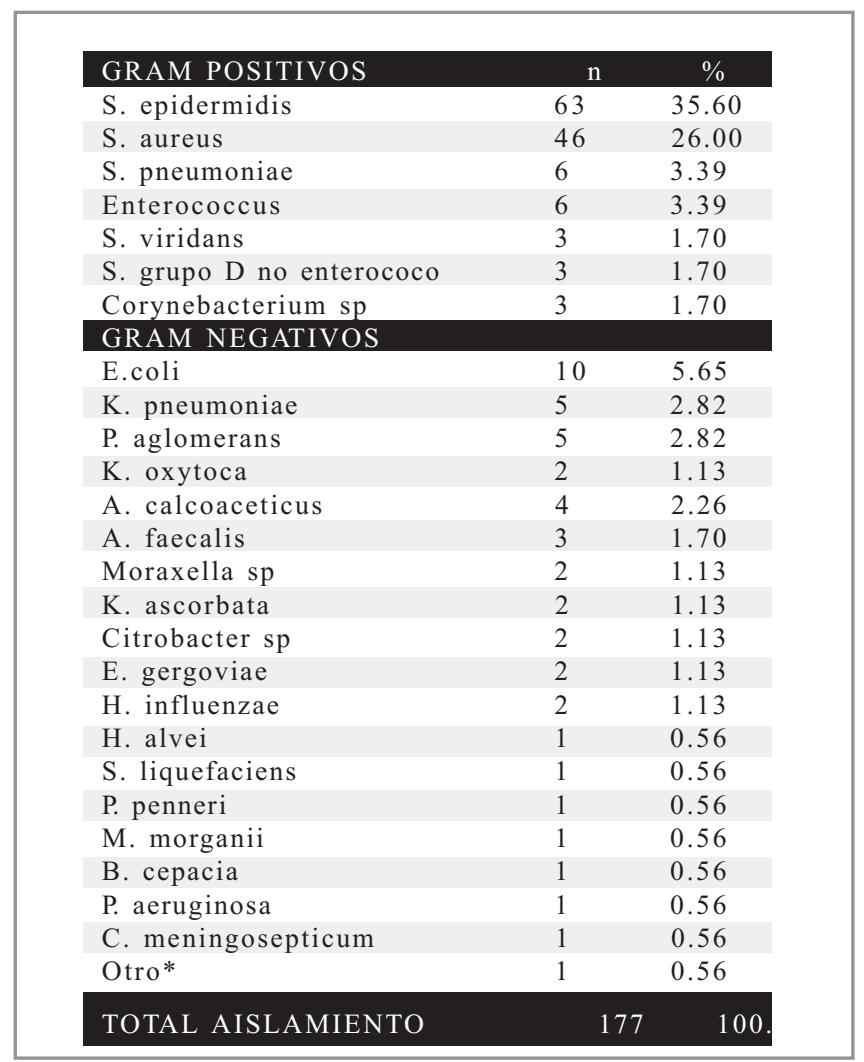

*Microorganismo aislado pero no identificado

Tabla 1. Frecuencia relativa (\%) de las especies bacterianas aisladas en 161 pacientes. 


\begin{tabular}{l|c} 
ESPECIES BACTERIANAS & CASO \\
AISLADAS EN UN MISMO PACIENTE & \\
\hline S. aureus - S. epidermidis & 4 \\
S. aureus - Enterococcus & 2 \\
S. epidermidis - S. pneumoniae & 2 \\
P. aglomerans - S. aureus & 1 \\
K. oxytoca - S. epidermidis & 1 \\
Citrobacter sp-S. aureus & 1 \\
H. influenzae-S. epidermidis & 1 \\
S. epidermidis - H. alvei & 1 \\
S. licuefaciens - S. epidermidis & 1 \\
Enterococcus - S. epidermidis & 1 \\
P. aeruginosa - C.meningosepticum & 1 \\
TOTAL & 16 \\
\hline
\end{tabular}

Tabla 2. Casos en los que se aislaron dos especies bacterianas a partir de la misma muestra.

enterococo $(4,11 \%)$. Utilizando el programa ESTATA 6.0 se pudo establecer que no existe asociación evidente entre la convivencia con animales y el resultado de aislamientos positivos para microorganismos de origen zoonótico.

De las especies bacterianas aisladas con el diagnóstico clínico, se observa que el mayor porcentaje de aislamientos fue realizado en pacientes con conjuntivitis bacteriana $(33,30 \%)$, blefaritis bacteriana (19,77\%), blefaritis inespecífica (18.64\%), conjuntivitis inespecífica $(11,29 \%)$, blefaroconjuntivitis $(6.21 \%)$, dacriocistitis $(4.52 \%)$, celulitis $(2,83 \%)$ y queratitis ulcerativa periférica en el $0.56 \%$ de los casos (Tabla 4 ).

\section{Discusión}

En esta investigación se obtuvieron 177 aislamientos encontrándose un notorio predominio de flora Gram-positiva 73.45\%. Las especies Grampositivas aisladas más frecuentes fueron: S. epidermidis $(48.46 \%)$, S. aureus $(35.38 \%)$, S. pneumoniae $(4.61 \%)$ y Corynebacterium sp. (2.31\%), que son consideradas flora habitual del ojo sano y que a menudo pueden causar infecciones externas como patógenos oportunistas cuando el tejido involucrado sufre algunos cambios en su estructura anatómica, su fisiología o su bioquímica; cambios que

\begin{tabular}{|c|c|c|c|c|}
\hline \multirow{2}{*}{ GRAM POSITIVOS } & \multicolumn{4}{|c|}{ CONVIVE CON ANIMALES } \\
\hline & SI & $\%$ & NO & \\
\hline S. epidermidis & 34 & 34.60 & 29 & 36.70 \\
\hline S. aureus & 24 & 24.40 & 22 & 27.80 \\
\hline Enterococcus & 4 & 4.08 & 2 & 2.50 \\
\hline S. pneumoniae & 4 & 4.08 & 2 & 2.50 \\
\hline S. viridans & 3 & 3.06 & 0 & 0.00 \\
\hline S. grupo D no enterococo & 3 & 3.06 & 0 & 0.00 \\
\hline Corynebacterium sp & 2 & 2.04 & 1 & 1.30 \\
\hline \multicolumn{5}{|l|}{ GRAM NEGATIVOS } \\
\hline E.coli & 7 & 7.14 & 3 & 3.80 \\
\hline K. pneumoniae & 2 & 2.04 & 3 & 3.80 \\
\hline K. oxytoca & 2 & 2.04 & 0 & 0.00 \\
\hline P. aglomerans & 3 & 3.06 & 2 & 2.50 \\
\hline K. ascorbata & 2 & 2.04 & 0 & 0.00 \\
\hline A. faecalis & 0 & 0.00 & 3 & 3.80 \\
\hline E. gergoviae & 0 & 0.00 & 2 & 2.50 \\
\hline H. alvei & 0 & 0.00 & 1 & 1.30 \\
\hline S. liquefaciens & 1 & 1.02 & 0 & 0.00 \\
\hline P. penneri & 0 & 0.00 & 1 & 1.30 \\
\hline M. morganii & 0 & 0.00 & 1 & 1.30 \\
\hline Citrobacter sp & 1 & 1.02 & 1 & 1.30 \\
\hline B. cepacia & 0 & 0.00 & 1 & 1.30 \\
\hline P. aeruginosa & 0 & 0.00 & 1 & 1.30 \\
\hline C. meningosepticum & 0 & 0.00 & 1 & 1.30 \\
\hline Moraxella sp & 1 & 1.02 & 1 & 1.30 \\
\hline H. influenzae & 2 & 2.04 & 0 & 0.00 \\
\hline A. calcoaceticus & 3 & 3.06 & 1 & 1.30 \\
\hline Otro* & 0 & 0.00 & 1 & 1.30 \\
\hline TOTAL & 98 & 55.37 & 79 & 44.63 \\
\hline
\end{tabular}

Tabla 3. Especies bacterianas aisladas asociadas con la convivencia o no con animales.

pueden ser inducidos, inclusive, por el mismo agente patógeno, favoreciendo su propia colonización y diseminación a tejidos adyacentes (8-10). Las especies identificadas en los pacientes como posibles agentes causales de la infección y su frecuencia de aislamiento concuerdan con los hallazgos de otros autores (11-13).

S. epidermidis y Corynebacterium sp comensales normales de conjuntiva y de párpado, al ser aislados por cultivo microbiológico podrían asociarse con contaminación, ya que los aislamientos son considerados clínicamente significativos si son consistentes con los signos clínicos, la observación microscópica y su crecimiento en más de uno de los medios de cultivo, o si se aislan en diferentes medios 


\begin{tabular}{|c|c|c|c|c|c|c|c|c|c|c|c|c|c|c|}
\hline \multicolumn{15}{|c|}{ GRAMPOSITIVAS } \\
\hline \multirow[t]{2}{*}{$\begin{array}{l}\text { DIAGNÓSTICO } \\
\text { CLÍNICO }\end{array}$} & \multicolumn{2}{|r|}{ 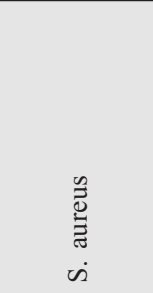 } & \multicolumn{2}{|c|}{$\begin{array}{l}\frac{n}{0} \\
\frac{0}{\Xi} \\
\frac{0}{0} \\
\frac{0}{0} \\
\dot{0} \\
\dot{0}\end{array}$} & \multicolumn{2}{|c|}{ 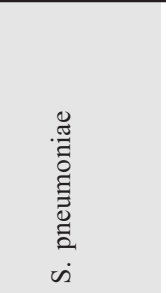 } & \multicolumn{2}{|c|}{ 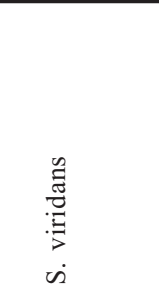 } & \multicolumn{2}{|c|}{ 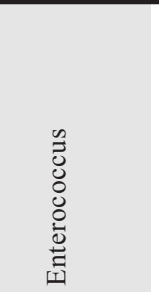 } & \multicolumn{2}{|c|}{ 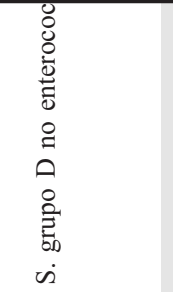 } & \multicolumn{2}{|c|}{ 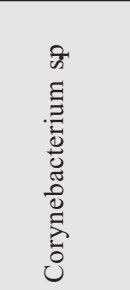 } \\
\hline & $\mathrm{n}$ & $\%$ & $\mathrm{n}$ & $\%$ & $\mathrm{n}$ & $\%$ & $\mathrm{~N}$ & $\%$ & $\mathrm{n}$ & $\%$ & $\mathrm{n}$ & $\%$ & $\mathrm{~N}$ & $\%$ \\
\hline Conjuntivitis bacteriana & 14 & 30.40 & 16 & 25.40 & 3 & 50.00 & 3 & 100 & 4 & 66.66 & 1 & 33.33 & 3 & 1 \\
\hline B. Bacteriana & 9 & 19.60 & 13 & 20.64 & 0 & 0 & 0 & 0 & 0 & 0 & 0 & 0 & 0 & 0 \\
\hline B. Inespecífica & 11 & 23.90 & 12 & 19.05 & 1 & 16.67 & 0 & 0 & 1 & 16.67 & 0 & 0 & 0 & 0 \\
\hline Conjuntivitis inespecífica & L. & 8.70 & 10 & 15.86 & 1 & 16.67 & 0 & 0 & 1 & 16.67 & 1 & 33.33 & 0 & 0 \\
\hline Blefaroconjuntivitis & 4 & 8.70 & 5 & 7.94 & 1 & 16.67 & 0 & 0 & 0 & 0 & 0 & 0 & 0 & 0 \\
\hline Dacriocistitis & 1 & 2.17 & 3 & 4.75 & 0 & 0 & 0 & 0 & 0 & 0 & 1 & 33.33 & 0 & 0 \\
\hline Celulitis & 1 & 2.17 & 2 & 3.18 & 0 & 0 & 0 & 0 & 0 & 0 & 0 & 0 & 0 & 0 \\
\hline Queratitis ulcerativa periférica & () & 0 & & 0 & 0 & 0 & 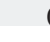 & 0 & & 0 & & 0 & & \\
\hline Otro & 2 & 4.35 & 2 & 3.18 & 0 & 0 & 0 & 0 & 0 & 0 & 0 & 0 & 0 & 0 \\
\hline TOTAL & 46 & 100 & 63 & 100 & 6 & 100 & 3 & 100 & & 100 & 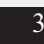 & 99.99 & & \\
\hline
\end{tabular}

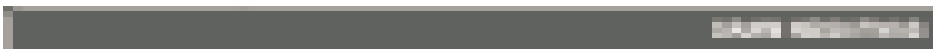

Tabla 4. Distribución del tipo de microorganismo aislado según el diagnóstico clínico (continúa en la siguiente página...)

(9). Las infecciones producidas por estos dos agentes habitualmente son localizadas pero pueden diseminarse desde la conjuntiva hasta la córnea, al ojo interno, a la órbita e incluso al cerebro $(12,14,15)$. En este estudio se encontraron tres casos de conjuntivitis bacteriana, dos en niños y una en adulto, cuyo agente etiológico aislado fue Corynebacterium sp.

S. epidermidis, reconocido componente de la flora conjuntival normal, actualmente es considerado patógeno oportunista, a menudo produce signos y 


\begin{tabular}{|c|c|c|c|c|c|c|c|c|c|c|c|c|c|c|c|c|c|c|}
\hline \multirow{3}{*}{$\begin{array}{l}\text { DMGNOSTICO } \\
\text { CLINICO }\end{array}$} & \multicolumn{18}{|c|}{ GRAM NEGATIMAS } \\
\hline & \multicolumn{2}{|c|}{$\begin{array}{l}\frac{3}{9} \\
\frac{3}{7} \\
\text { b } \\
\text { r }\end{array}$} & \multicolumn{2}{|c|}{ 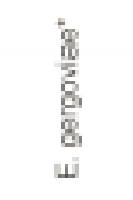 } & \multicolumn{2}{|c|}{$\frac{\frac{\pi}{\frac{D}{4}}}{\frac{\partial}{4}}$} & \multicolumn{2}{|c|}{$\begin{array}{l}\frac{\mathrm{E}}{\mathrm{E}} \\
\mathrm{E} \\
\mathrm{I}\end{array}$} & \multicolumn{2}{|c|}{ 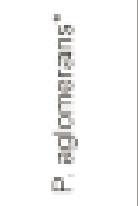 } & \multicolumn{2}{|c|}{$\begin{array}{l}\frac{1}{\mathrm{I}} \\
\frac{\mathrm{y}}{\mathrm{n}} \\
\mathrm{I}\end{array}$} & \multicolumn{2}{|c|}{ 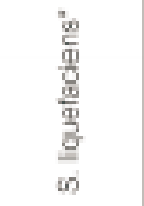 } & \multicolumn{2}{|c|}{$\frac{8}{8}$} & \multicolumn{2}{|c|}{$\frac{1}{8}$} \\
\hline & n & 8 & n & S & n & $\%$ & n & $y$ & $\mathbf{n}$ & $y$ & $\mathrm{n}$ & $\%$ & $\pi$ & $\%$ & n & $\mathrm{K}$ & n & $\%$ \\
\hline $\begin{array}{l}\text { Cenyuntivitis } \\
\text { bocteriana }\end{array}$ & 1 & 50 & 1 & 60 & 1 & 100 & 0 & 0 & 0 & 0 & 0 & 0 & 0 & 0 & 0 & 0 & 69 & 35.3 \\
\hline B. Bacterisns & 0 & 0 & 0 & 0 & 0 & 0 & 0 & 0 & 4 & 89 & 1 & 100 & 1 & 100 & 0 & 0 & 35 & 19.77 \\
\hline B Inespos fica & 0 & 0 & 1 & 50 & 0 & a & a & $a$ & 1 & 20 & 0 & 0 & 0 & 0 & 1 & 100 & 33 & 18.44 \\
\hline $\begin{array}{l}\text { Conjuntivitis } \\
\text { inespers tha }\end{array}$ & D & 0 & 0 & 0 & 0 & ] & a & 0 & 0 & 0 & 0 & 0 & $\square$ & D & 0 & 0 & 20 & 1129 \\
\hline Elefaroconjuntrites & 0 & 0 & 0 & 0 & 0 & 0 & 1 & 100 & 0 & 0 & 0 & 0 & 0 & 0 & 0 & 0 & 11 & 621 \\
\hline Dacriacistitis & 1 & 50 & 0 & 0 & 0 & 0 & 0 & 0 & 0 & 0 & 0 & 0 & 0 & 0 & 0 & 0 & 8 & 452 \\
\hline Celulitis & 0 & 0 & 0 & 0 & 0 & 0 & 0 & 0 & 0 & 0 & 0 & 0 & 0 & 0 & 0 & 0 & 5 & 283 \\
\hline $\begin{array}{l}\text { Queratitis uberativa } \\
\text { pent rea }\end{array}$ & D & 0 & 0 & 0 & 0 & 0 & 0 & a & 0 & 0 & 0 & 0 & 0 & 0 & 0 & 0 & 1 & 0 58 \\
\hline Giro & 0 & 0 & 0 & 0 & 0 & 0 & 0 & 0 & 0 & 0 & 0 & 0 & 0 & 0 & 0 & 0 & 5 & 283 \\
\hline TOTAL & 2 & 100 & 2 & 100 & 1 & 100 & 1 & 100 & 5 & 100 & 1 & 100 & 1 & 100 & 1 & 100 & $17 T$ & 100 \\
\hline
\end{tabular}

Tabla 4. Distribución del tipo de microorganismo aislado según el diagnóstico clínico (continuación).

síntomas típicos de blefaroconjuntivitis Staphylococcica crónica (15). En el presente estudio, S. epidermidis fue aislado en un $25.40 \%$ de las conjuntivitis, $20.64 \%$ de las blefaritis, $7.94 \%$ de las blefaroconjuntivitis y en un $15.86 \%$ y $19.05 \%$ de las conjuntivitis y Blefaritis inespecíficas respectivamente. La alta frecuencia de los hallazgos de Staphylococcus sp. en la blefaritis es el resultado de la ocurrencia de una conjuntivitis previa por el mismo microorganismo (9).

S. aureus aislado en segundo lugar de frecuencia en las infecciones oculares externas, diagnosticadas clínicamente como bacterianas, también ha sido reportado por otros investigadores como causa común de conjuntivitis y blefaritis bacteriana (16-17). Los hallazgos en este estudio muestran una frecuencia de aislamiento en un $30.40 \%$ de las conjuntivitis y un $19.6 \%$ de las blefaritis, además en un porcentaje significativo (23.9\%) de las blefaritis inespecíficas.
Otros microorganismos que son menos comunes pero que pueden transitoriamente ser parte de la flora normal y convertirse en patógenos oportunistas son S. viridans (2 a $6 \%$ ) y S. pneumoniae (1 a $5 \%$ ) (10). En este estudio, el porcentaje de aislamientos de $\mathrm{S}$. viridans y S. pneumoniae fue del 2,36\% y 4,72\% respectivamente, del total de aislamientos de bacterias Gram positivas.

El 26.55\% del total de aislamientos correspondió a bacilos Gram negativos, de los cuales el $74.47 \%$ son enterobacterias y el $25.53 \%$ microorganismos no fermentadores. Estos resultados coinciden con lo reportado en la literatura (18).

Se aisló E. coli de diez muestras así: tres casos de conjuntivitis bacteriana, dos de blefaritis bacteriana, dos de conjuntivitis inespecífica, uno de dacriocistitis, queratoconjuntivitis y blefaritis inespecífica respectivamente. La E. coli generalmente no causa infección ocular; sin embargo, puede hallarse en bajo por- 
centaje en conjuntivas normales, especialmente en personas con problemas intestinales o luego de un uso prolongado de antibióticos. Se ha descrito a nivel de conjuntiva como una oftalmía purulenta neonatal, también en conjuntivitis pseudomembranosa en adultos y, en un mínimo porcentaje, en queratitis con hipopión, dacriocistitis y celulitis orbital (19).

Las especies M. morganii y P. penneri fueron aisladas de un caso de blefaritis inespecífica y blefaroconjuntivitis respectivamente. Estos microorganismos son considerados invasores secundarios involucrados en queratitis severa, similar a la producida por Pseudomona sp. pero con un proceso más rápido y destructivo que puede llevar a perforación de la córnea (13). La Pseudomona aeruginosa es un patógeno común aislado de celulitis orbitaria, conjuntivitis membranosa secundaria a queratitis que puede cursar rápidamente con destrucción corneal y necrótica teniendo como factor de riesgo una lesión corneal con cuerpo extraño $(13,8,9)$. En este estudio se aisló una P. aeruginosa asociada a celulitis.

Otro microorganismo aislado de un paciente con conjuntivitis bacteriana que presentó cuerpo extraño y además usaba lentes de contacto fue Burkholderia cepacia perteneciente a la familia Pseudomonadaceae. Koneman, et al.(20) describen a B. cepacia, reconocido fitopatógeno que ocasionalmente se ha recuperado de casos de pacientes con enfermedad fibroquística y conjuntivitis bacteriana; además, la enfermedad ocular generalmente se presenta con antecedentes de lesión corneal, especialmente por un cuerpo extraño.

En el presente trabajo se aislaron microorganismos, hasta ahora sin reporte previo en literatura, como causantes de infección ocular externa; a nivel de bacterias Gram positivas se aisló Enterococcus y S. Grupo D no entorococo; en cuanto a las Gram negativas se identificó Alcaligenes faecalis, Citrobacter $\mathrm{sp}$,Chryseobacterium meningosepticum y siete especies diferentes de la familia Klebsielleae; por lo tanto, es importante llamar la atención sobre su aislamiento en la población de estudio debido a que su casual aislamiento como agente etiológico puede obedecer a la capacidad de establecerse como patógeno oportunista $(21,22)$. Es probable que al incrementar el cultivo microbiológico en el diagnóstico de infecciones oculares, estos aislamientos sean más frecuentes y se pueda determinar su implicación definitiva como patógeno ocular.

El contagio del ojo por patógenos microbianos depende del contacto directo $\mathrm{y}$, en gran parte, de las condiciones de salubridad e higiene del paciente, así como del contacto con personas o animales portadores de microorganismos. La frecuente relación entre humanos y animales favorece el intercambio de agentes infecciosos entre las especies. Dentro de las bacterias reportadas en la literatura que han generado infecciones oculares y que están asociadas con animales se encuentran: Brucella, Leptospira, Salmonella spp, Francisella tularensis, Yersinia pestis, Pasteurella multocida, Chamydia psittacii, Streptococcus y Staphylococcus $(23,6,7,4)$. En este estudio se aislaron especies de Streptococcusy Staphylococcus, pero no se encontró evidencia significativa entre la presencia de estas bacterias en infecciones oculares y la convivencia o no con animales. Igualmente, esta investigación no mostró una relación significativa al comparar las variables convivencia o no con animales y tipo de microorganismo aislado asociado a zoonosis (Tabla 3 ).

\section{REFERENCIAS}

1. O’Brien TP, Hazlett L. Ocular Microbiology: Pathogenesis of ocular infection; 2000.

2. Ang T, Khan PK. Nossocomial Klebsiella pneumoniae conjunctivitis resulting in infections keratitis ambilateral corneal perforation. Arch Ophthalmology 1996 Apr;114(4):933-6.

2. Walker S. Microbiología. México: Mc Graw-Hill Interamericana; 2000.

3. Dissanaike AS, Jayaweera CD, Padmini HH, Ihalamulla RL, Naotunne T. Recovery of a species of Brugia, probably B.

www.unicolmayor.edu.co 
ceylonensis from the conjuntiva of a patient in Sri Lanka. Ann Tropical Med \& Parasitol 2000;94(1):83-6.

4. Knoor HL, Weber A. Ocular manifestations of selected zoonoses in human. Tierarztl Prax 1992;20(4):347-54.

5. Rathinam S, Cor E, Alex JC, et al. Leptospiral antibodies among human beings in Tamilnadu. Indian J Med Microbiol 1993;11:203-5.

6. Falck G. Group A streptococci in household pets eye a source of infection in humans. Scand J Infect Dis 1997;29(5):46971.

7. Glickman LT, Magnaval JF. Zoonotic roundworm infections. Infect Dis Clin North Am 1993;7(3):717-32.

8. Marlin D. Conjunctivitis, Bacterial. Hospital Los Angeles Medical Center, Health Science Center and University of Arkansas for Medical Science; 2001 Aug: 4-9.

9. Bruce Jackson W. Diagnosis and treatment eye. The Ottawa Hospital, Eye Institute; 2001.

10. Syed NA, Hyndiuk RA. Infectious Conjunctivitis. Infectious Disease Clinics of North America; 1992 Dic;6(4):789-805.

11. Niranjan N, Gita S. Slime production as a virulence factor in Staphylococcus epidermidis islated from bacterial keratitis. Indian Journal of Medical Research 2000 Jan;111:6-11.

12. García-Saenz MC, Peral Ortiz de la Torre MJ, De Castro LM, Jiménez Martínez E, García Sánchez JE, Fresnadillo MJ. Flora conjuntival según edades. Archivos Sociedad Española de Oftalmología; 1999 Jul;(7):1-7.

13. Schaefer F, Bruttin O, Zografos L, Guex-Crosier Y. A prospective clinical and microbiological study. British Journal of Ophthalmology $2001 \mathrm{Jul} ; 85(7): 842-8$.
14. Otto S, Aydin P, Cifetciogu N, Dursun D. Slime production by coagulase-negative staphylococci isolated in chronic blepharitis. Eur J Ophthalmol 1998;8(1):1-3

15. Srinivasan M, González A, Celine G, Cevallos V. Epidemiology and aetiological diagnosis of corneal ulceration in madural, South India. British Journal of Ophthalmology 1997 Nov;29(11):965-72.

16. Scott UI, Flynn HW, Feuer W, Pflugfelder, Forster RK, Miller D. Endophthalmitis associated with microbial keratitis. Ophthalmology 1996 Nov;103(11):1864-70.

17. Modarres SH, Lashell A, Oskoii N. Bacterial etiologic of ocular infection in children in the Islamic republic of Iran. Ocular infection; $4(1): 44-49$

18. Dennis P. Hans and the Endophthalmitis Vitrectomy Study Group. Microbiologic Factor and Visual Outcome in the Endophthalmitis Vitrectomy Study. American Journal of Ophthalmology 1996 Dec;122:830-46.

19. Mezer E, Gelsand YA, Lottan R, Tamir A, Miller B. Bacteriological profil of ophthalmology infection in and Israeli Hospital. Eur-J-ophtalmol 1999 Apr- Jul;9(2):120-4.

20. Koneman EW, et al. Diagnóstico Microbiológico. Brasil: Ed. Panamericana, 2000.

22. Reynolds HY. Pneumonia due to Klebsiella. In: Wyngaarden JB, Smith LH, editors. Cecil textbook of Medicine. 16th ed. Philadelphia; 1982.

23. Rathinam SR, Rathnam S, Suresh B, et al. Leptospiral antibodies in patients with recurrent ophthalmic involvement. Indian J Med Res 1996;103:66-8. 